\title{
The Dynamic Nature of Availability
}

\author{
Agnieszka Szóstek ${ }^{*}, 1$, Panos Markopoulos ${ }^{2}$ and Berry Eggen ${ }^{2}$ \\ ${ }^{1}$ Institute of Information Processing, Al. Niepodległości 188b, 00-608 Warsaw, Poland \\ ${ }^{2}$ Industrial Design Department, Eindhoven University of Technology, Den Dolech 2, 5600MB Eindhoven, The \\ Netherlands
}

\begin{abstract}
The topic of availability management has been extensively investigated in related research. However, thus far the approach to this subject was primarily motivated by the need to protect an interruptee from an unwanted interruption. In this approach, availability status was assumed to be static: either the interruptee was interactive, thus willing to accept an interruption or interpassive, thus prone to reject it. In this research we would like to propose a different assumption: that availability status has a dynamic rather than a static nature and that both communicators conjointly influence that status. To test that assumption, we explored the nature of availability and factors that are likely to influence it through a series of empirical investigations. These studies have shown that availability state is likely to be influenced by factors such as: social proximity, nature of the communication subject and anticipated interruption duration. We have also observed that while social proximity was shown to be a crucial factor for face-to-face communications, it appeared to have little impact on the availability status in email communications.
\end{abstract}

Keywords: availability, face-to-face communication, email communication, awareness, communication systems, computer supported collaborative work.

\section{INTRODUCTION AND RELATED WORK}

Informal communication in the workplace has been described as an essential mechanism for effective work [1]. Such communication is often born through an opportunistic attempt of one person (an interruptor) to initiate a communicative exchange that often causes an interruption of another person's (an interruptee's) ongoing activity. Nowadays, people use a variety of communication means (also referred to as communication channels) to support information sharing and contact with others. A communication channel can be defined as a line of communication that is used to transmit information from a sender to a recipient and it can be rooted in either the physical or the digital domain. The channel selected to communicate plays an integral role in the context and the process of a communicative exchange [2-4]. Basically, any decision behind channel selection can be seen as an optimization process: people consider which channel helps them best express their intentions and needs [5]. Richer media such as face-to-face or phone are more likely to be chosen to deal with potentially complex and ambiguous tasks such as conflict solving or decision taking [6]. Less rich media, such as email or Instant Messaging, are more likely to be employed for resolving simple tasks like scheduling or confirming earlier agreements.

In order to establish any communication both communicators first enter an interruption negotiation process during which a recipient needs to assess what are the expectations

*Address correspondence to this author at the Institute of Information Processing, Al. Niepodległości 188b, 00-608 Warsaw, Poland; Tel: +48222124053; Fax: +48228253319;

E-mail: aga.szostek@opi.org.pl of an initiator [7-9] and the initiator has to interpret signs indicating whether the moment is appropriate to initiate communication [10-12]. In this negotiation process the recipient has usually a choice to become interactive -immediately engage in the communication or interpassive -decide not to engage in the communication at that particular moment $[13,14]$. Such a decision has been found to depend on the recipient's availability but is also likely to be influenced, for example, by the subject of the interruption [15-18]. One may be willing to immediately accept an interruption that helps one's progress with one's primary task or when it contains information one has been waiting for [12]. Therefore, we set out to understand what is the nature of availability and what factors are likely to have an impact on the decision of the interruptee about how to deal with an interruption. More specifically, we set out to compare two channels: face-to-face and email communication. The main differences between these two channels pertain to their richness, which relates to the variety of signals that are possible to be shared between communicators [8, 11, 19]. Both channels further differ in the temporal dimension, namely the synchronous or not nature of the channel, which affects pacing of communication according to the needs and desires of both communicators [20-22]. Finally, both channels offer different levels of control the initiator and the recipient have over the communicative occurrence, which is substantiated in the possibility for them to choose when and how to react to communication initiation $[14,23]$.

This research introduces the notion of communication negotiation as a part of communication process (see: Fig. 1). In this negotiation the recipient needs to assess the expectations of the initiator $[7,8]$ and the initiator has to interpret signs indicating if the moment to communicate is appropriate [10-12]. For any communication to be 
successful, both communicators need to reach an agreement on how to cope with its content within the given time limitations [24]. An adequate behaviour is often motivated by the social and professional relationship between the actors $[8,25]$. Such a behaviour is also contingent upon aspects such as recipient's own time-pressure or the next activity planned [7, 11, 18, 26-28]. In the related literature, such communication negotiation is often referred to as an interruption and introduces the notion of availability management as a means to determine an appropriate moment of communication initiation [18, 29-31].

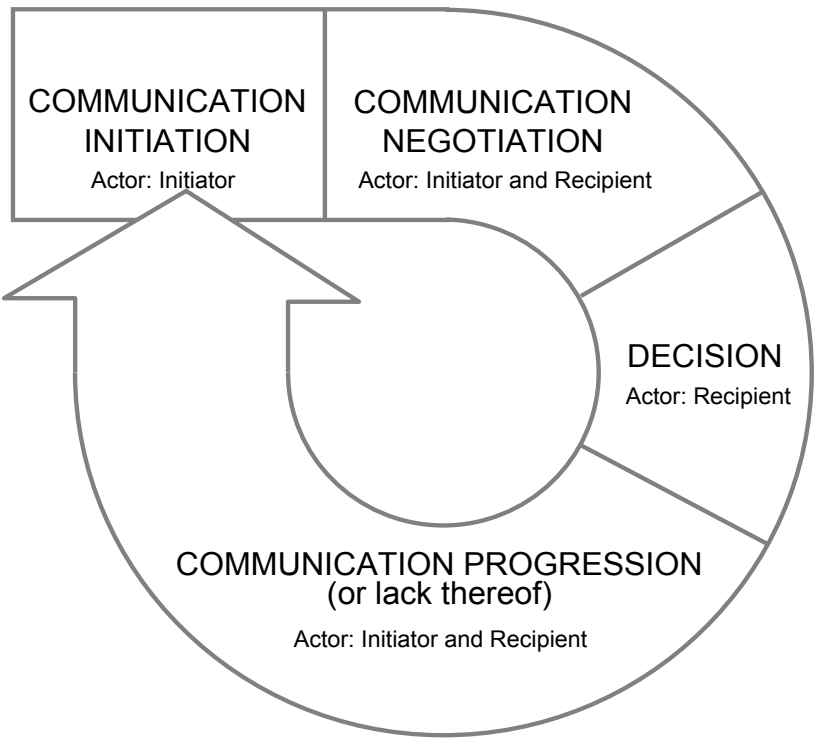

Fig. (1). The model of the communication process. The research described in this article focuses on the first phase of that process where the communicators negotiate their communicative contract.

To answer the research question, an series of studies was conducted. We began with an observational study of how administrative assistants assess face-to-face interruptions. Next, semi-structured interviews with twelve knowledge workers were conducted that aimed to better understand the dependencies among different factors influencing their interruption behaviours. Both studies focused on face-to-face communication that is considered the richest communication channel and at the same time imposing the heaviest burden caused by a communicative attempt in terms of resumption lag to the primary task of the interruptee $[32,33]$ and also high social cost for both communicators [8, 34, 35]. Therefore, as the last step, we conducted a diary study that assessed the impact of the identified factors on the decision how to handle emails.

\section{ADMINISTRATIVE ASSISTANTS AND HANDLING INTERRUPTIONS}

The work of administrative assistants has not been extensively studied within the $\mathrm{CHI}$ and $\mathrm{CSCW}$ domains. A recent study by Erickson et al. [36] aimed to capture the complexity of assistants' job. The authors showed that maintenance of situational awareness, the continuous use of extensive background knowledge regarding the organization and its processes and also collaborative skills well describe assistants' responsibilities. As part of their daily tasks, assistants are also professional interruption mediators. They use a sophisticated set of own heuristics to assess and negotiate interruptions both for their managers but also for themselves. Interruption negotiation methods of assistants could provide a great source of information to better understand what factors influence the decision about how to handle interruptions and consequently guide related attempts to create systems supporting mediated communication.

Dabbish and Baker [16] investigated strategies assistants applied for negotiating interruptions using semi-structured contextual interviews [37]. They identified two factors which are central to deciding whether to allow or disapprove an interruption: importance of the interruptor and importance of the interruption subject. According to their model the calculation of the importance of the subject seemed to be a combination of how important the problem at hand was to the interruptor ${ }^{1}$ combined with the importance of the interruptor as a person. Individuals of low importance tended to be asked what their business was, whereas individuals of high importance were given immediate access whenever possible. Their study, however, derived its results from participants' retrospective accounts rather than trying to capture their momentary reflections within a specific situation of interruption handling [38]. The first study was set out to verify the model of Dabbish and Baker [16] through an observational study that aimed at capturing the richness of the interruption handling process by analyzing interruption events at the moment of their occurrence. This method should allow to identify what other aspects influence the change in the availability state of the assistant and in consequence the interruption outcome, e.g. urgency [31,32] or social costs $[34,39]$.

\subsection{Study Design and Analysis}

The study examined how interruptions were handled by three administrative assistants (two from an academic and one from an industrial environment). All assistants were female and had more than 10 years of working experience at similar positions. They all managed the schedule of their bosses, handled communications by mail and phone and receive visitors. They also dealt with issues of other employees of their work groups.

Observations and interviews were conducted in-situ and concerned both interruptions directed at the assistants and at their managers. Assistants were observed for a day each as they dealt with interruptions. The observer monitored all incoming interruptions and coded them according to the following criteria:

- Importance of the interruptor (based upon an organizational chart).

- Urgency of the interruption subject assessed by the assistant.

- Importance of the interruption subject assessed by the assistant.

- Assistant's availability level (coupled to the performed task).

\footnotetext{
${ }^{1}$ We distinguish in this text the role of the interruptor, i.e., the initiator of communication, and the interruptee, i.e. the recipient of the communicative request.
} 
- Interruption outcome (immediately handled/postponed).

All there criteria reflected the criteria for assessing interruptions identified by Dabbish and Baker [16]. The observer attempted to assess each interruption as completely as possible just by observing the event. Whenever in doubt, she requested clarification from the assistant after the interruption was concluded to avoid influencing the assistant's behaviour. Observations that were difficult to classify were redirected to the assistant in a form of the following open question: 'How would you describe this interruption?', followed by closed questions asked according to the above described criteria whenever the answer provided by the assistant did not render adequate information. We chose for capturing an immediate reaction (rather than, for example, video-taping the interactions and discussing them later) to avoid rationalizing or simply forgetting the decisions behind participant's reactions.

All interruptions were recorded in a spreadsheet and given a unique identifier. In addition, each interruptor was asked to fill in a brief questionnaire (see: Fig. 2) printed on a card with two items reported on a 5-point Likert scale and concerning:

- Urgency of the interruption subject (which was operationalized by assessing the time frame, within which the interruption should be handled),
- Importance of the interruption subject (which was operationalized by assessing how important the interruption subject was for the interruptor).

The cards were then entered into a sealed box, which only the observer had access to. Questionnaire cards could be linked with the adequate spreadsheet records through a unique identifier.

At the end of each observation day semi-structured interviews were conducted. All interruptions of the study day were revisited and questions regarded ways of handling interruptions, factors influencing the evaluation of each interruption and strategies for screening them. Assistants were presented with the spreadsheets containing the data collected during the day so that they were able to base their reflections on the actual events. Each interview lasted about 60 minutes and was audio-recorded for further analysis.

The data collected during the observations was first checked for completeness. A data point was considered as complete when it contained a depiction of the observed assistant's behaviour linked to the impression about that interruption described by the interruptor. In such a way, a set of 48 data points was gathered. Next, the causal variables (the importance of the interruptor, urgency and importance

\title{
TU/e department of \\ industrial design
}

\author{
Please mark your answers \\ and put the questionnaire into the ballot box located outside the office:
}

1. How fast did you want your problem to be handled?

- Whenever

- This week

- Today

- In the next 2 hours

- Immediately

2. How important was the interruption subject for you?

- Unimportant

- Rather unimportant

- Not so important

- Important

- Very important

Fig. (2). The questionnaire card provided to the interruptors arriving at the assistant's office. 
of the interruption subject, and availability of the assistant) were coded in the following way:

- Interruptors marked on the 5-point scale as either very important or important were coded as important, while those marked as equally important, less important or unimportant were coded as unimportant.

- Interruptions marked on the 5-point scale as either very important or important were coded as important, while those marked as equally important, less important or unimportant were coded as unimportant.

- Interruptions marked on the 5-point scale as either to be immediately handled or to be handled in the next 2 hours were coded as urgent, while those marked as to be handled today, this week or whenever were coded as not urgent.

- $\quad$ Availability state of the assistant marked on the 5point scale as either available or rather available were coded as available, while those marked as to be slightly unavailable, rather unavailable or unavailable were coded as unavailable.

Then, the binomial values together with the confidence intervals were separately calculated for each of the causal variables and the dependent variables (i.e., interruption outcome) [40]. Finally, a comparison of median values was made for the differences in perception of interruption importance and urgency between the assistants and the interruptors.

Then the interview recordings were transcribed and each statement was summarized on a post-it note. Next, a qualitative analysis of the transcripts was conducted, including open-coding (where categories emerged from the data rather than being defined a priori) [41] and card sorting with affinity diagrams to let higher order relationships between these categories to be defined [37, 42].

\subsection{Results}

A number of 48 interruption events handled by the assistants (21 interruptions were handled by assistant 1,15 by assistant 2 and 12 by assistant 3 ) were observed in the study. A number of 44 interruptions was intended directly for the assistants and 4 intended for their bosses, for which assistants acted as gatekeepers. Among these interruptions, 26 immediately handled occurrences were observed, 9 that were postponed and 13 occurrences that were diverted either to another person or back to the interruptor.

\subsubsection{Importance of the Initiator}

Assistants showed to have a clear distinction regarding the differences in the importance of the interruptor. Other managers and external guests were considered to be important interruptors. Members of the group managed by the manager the assistant was working for and also other assistants were considered as holding a similar position to that of the assistant. Students and support staff such as cleaning crew were considered as organizationally less important. The interruptions observed in the study, which were aimed directly at the assistants, were in the majority of cases initiated by interruptors holding a position that was considered similar in organizational importance to that of the assistant. All four interruptions aimed at the managers, for which assistants acted as gate-keepers, were initiated by important interruptors.

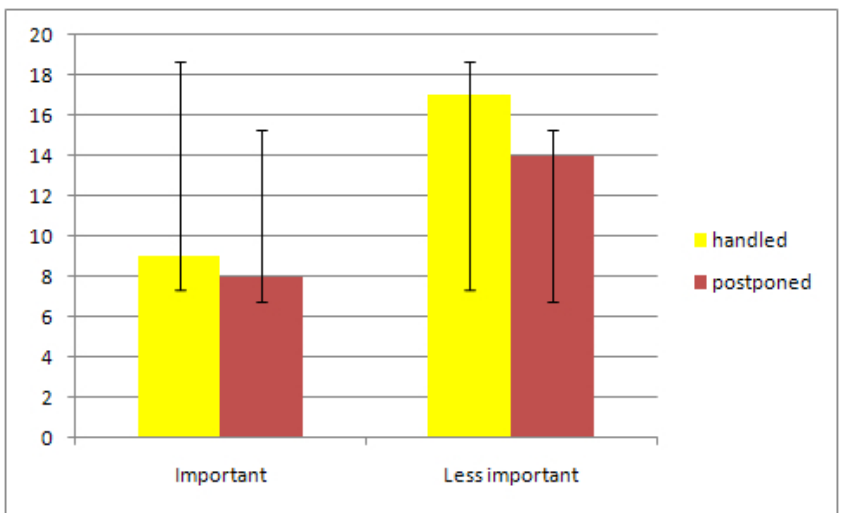

Fig. (3). The probability regarding the influence of the importance of the interruptor on the assistant's decision how to handle an interruption.

It was observed (see: Fig. 3) that interruptions initiated by people of equal or lower organizational status and those initiated by people with higher status had a similar chance to be immediately handled or to be postponed. These results imply that importance of the interruptor cannot be seen as a reliable predictor of the assistant's interruption behaviour.

Two strategies for handling interruptions by the assistants could be further distinguished; one for managing interruptions aimed at managers and another for interruptions aimed directly at them. In the first case, they did not ask an important person about the problem but allowed an interruption providing that the manager was available. If the manager was busy, the assistant would interrupt in an appropriate manner and negotiate an apt moment for handling the interruption. When an important person came to interrupt them, assistants would always inquire for the reason and then prioritize the problem according to its urgency, estimated time needed to deal with it and their own availability.

\subsubsection{Importance and Urgency of the Interruption Subject}

This study went beyond the observations of Dabbish and Baker in noting that, next to the importance of the interruption subject, also its urgency showed to play an important role when deciding on how to handle interruptions. The collected data suggested that interruptions considered as urgent, important or both had a higher probability to be immediately handled than to be postponed (see: Fig. 4).

Moreover, the data analysis showed that the perception of the importance of the interruption subject appeared to be equal for both assistants and interruptors (median $($ ImportanceInterruptor $)=4 ;$ median $($ ImportanceAssistant $)=$ 3.5). Among the interruptions identified as equally important were direct orders from the manager or urgent problems of employees. No difference with respect to the interruption handling behaviour between the industrial and the academic environment was observed.

A particular type of interruptions was eminent in this study: confirmative interruptions. Interruptors dropped by the assistant's office to check whether an earlier request had 
been taken care of, e.g., whether a hotel booking for an expected guest had already been arranged. In such a way interruptors tried to convey the urgency of the issue at hand through their physical presence or receive acknowledgements regarding the status of the task at hand.

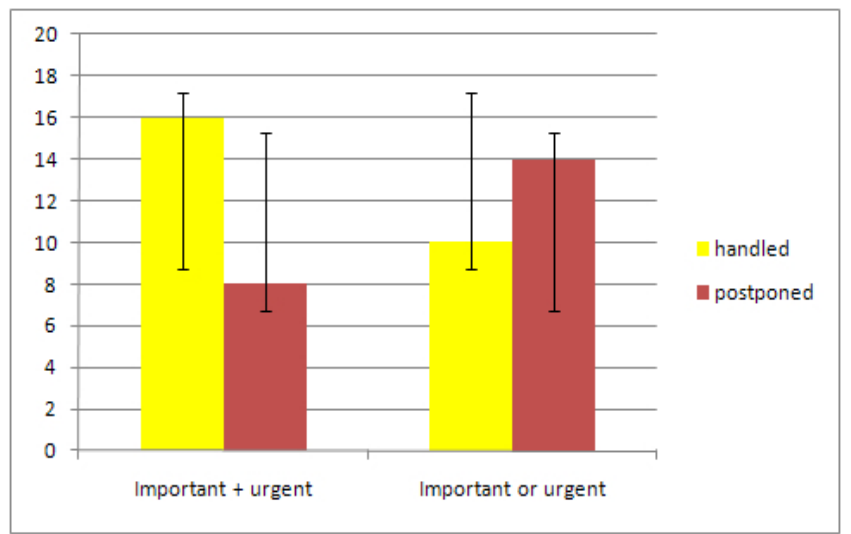

Fig. (4). The relative impact of the urgency and importance of the interruption subject on the assistant's decision how to handle an interruption.

\subsubsection{Availability of the Assistant}

In general, assistants considered themselves as generally available for interruptions (median $=4$ ). The collected data showed that an interruption could be equally probably immediately handled as postponed in the situation when the assistant was available (see: Fig. 5). Interestingly, it was noted that an interruption had a higher probability to be immediately handled when the assistant considered him or herself unavailable (though the difference is not significant).

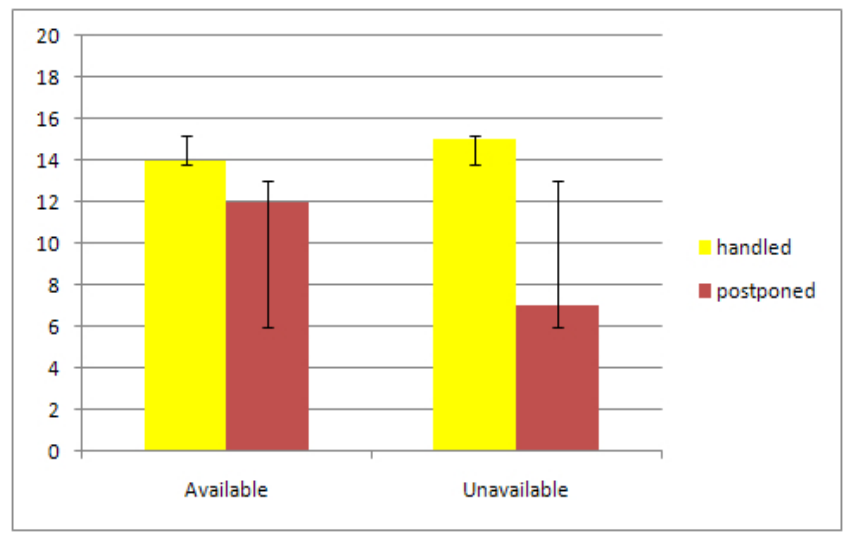

Fig. (5). The relative impact of the influence of assistant's availability on his or her decision how to handle an interruption.

Not a single interruption was perceived as annoying by the assistants. All participants commented during the postobservational interviews that they consider dealing with interruptions as part of their professional responsibilities and that they derive personal satisfaction from helping others to solve work-related problems. Assistants also stated that, in the majority of cases, it was not difficult for them to recover from the interruption so it seemed that the cost of an interruption was relatively low compared to that reported for knowledge workers [7, 26, 33, 43-45].

\subsection{Discussion}

This study evaluated and extended the model proposed by Dabbish and Baker that aimed at establishing factors influencing the decision of an assistant regarding an interruption outcome [16]. The results showed that an interruption considered as important, urgent, or both, had a high probability to be immediately handled regardless of how busy the assistant was. Interestingly, an interruption has a higher probability to be immediately handled at moments when the assistant considered him or herself unavailable. It could be explained by the fact that, in the situations of unavailability, assistants preferred to deal with the interruptions right away as a way to avoid having to remember about it later on. The interviews also revealed that many interruptors used physical presence as an indication of the increased urgency of the interruption and also means to remind the assistant about some problem that needed to be dealt with (e.g., booking a flight for an upcoming business trip).

Contradictory to the results reported by Dabbish and Baker [16], no positive impact of the importance of the interruptor on the interruption outcome was observed. Assistants seemed to equally often immediately handle as to postpone interruptions coming from both the important and less important people from their organization. From the interview data, it could be seen that importance of the interruptor seemed to play a role only to interruptions aimed at managers, where assistants acted as gatekeepers. This result, however, would have to be further confirmed as only a limited number of instances of interruptions aimed at the managers was detected. For interruptions aimed directly at the assistants, a different strategy was applied: the assistant would then find out first the importance and the urgency of the problem and subsequently prioritize according to these two factors. The importance of the interruptor seemed to have a lesser value in that process. This result might be, however, biased by the fact that the study was conducted in The Netherlands, where the power distance at work tends to be low. In other cultures with a stronger hierarchical structure this situation might be different and the professional position of the interruptor could prove to have a larger impact on the interruption negotiation process. Finally, a new determinant for interruption behaviours emerged in this study. The analysis of the qualitative data collected during the post-observational interviews showed that short interruptions seemed to have a higher chance to be immediately handled comparing to these requiring larger amount of time. The possible impact of this factor needed yet to be confirmed by future research.

In the conclusion of this study, we noted that, although assistants could be perceived as most capable to analyze the relevance of interruptions at work, they may not be the optimal subjects for assessing the interplay of different factors and potential change in the availability status of the interruptee. Assistants appeared to be, generally, available for interruptions at any time. They also showed a very positive attitude towards the incoming interruptions regardless of their own availability level. They considered it 
a part of their job to be interruption driven and derived a job satisfaction from helping others resolving their problems. Due to such an attitude they often found themselves in a weaker position compared to the interruptor as they did not want to mistreat a person who took an effort to personally come to their office. While assisting others is a primary task for assistants the nature of the work of knowledge workers is radically different. Handling interruptions is a distraction rather than a main task. Therefore, as a next step we set out to investigate the interruption handling practices employed by this user group. Our focus was two-fold: (i) to better understand the nature of knowledge workers' availability status and (ii) to further explore the relative influence factors such as urgency and importance of the interruption subject, the importance of the interruptor and also interruption timedemand could have on that status.

\section{KNOWLEDGE WORKERS AND HANDLING INTERRUPTIONS}

Prior research pointed at the fact that in face-to-face communication an interruptee has less control over communication compared to the interruptor $[14,23,46]$ and also pays the higher price in terms of the resumption lag, information overload and increased level of stress [7, 27, 28, $33,45$,$] . Therefore, when analyzing interruption behaviours$ researchers tend to take the interruptee's perspective on interruption negotiation $[24,31,43]$ and consider availability as the best predictor of an appropriate interruption moment [47]. However, the previous study showed that availability can be altered by factors such as, for example, importance or urgency of the communication subject. This study regarded availability management and interruption handling practices of knowledge workers. Exploring these differences aimed to better understand which information needs have to be addressed to leverage social behaviours in mediated communication.

Once more face-to-face communications were examined, as they are considered the richest communication channel [23] and therefore most likely to derive a comprehensive set of factors influencing ways interruptions are handled. This time the study took the form of semi-structured contextual interviews to help obtaining rich accounts of participants' perceptions regarding their interruption behaviours. It is important to note that, when people report on their experiences, the experiences themselves should be accessible to introspection allowing for accurate reports [48]. When the report reflects a recent episode, people draw on their episodic memory, retrieving specific details of the recent past [38, 49]. Likewise, global reports of past experiences are based on semantic knowledge. When asked how they 'usually' experience a particular activity, people tend to draw on their general beliefs and their attributes. The actual experience does not play a vital role anymore in that report as it is no longer available to the introspection and episodic reconstruction. Therefore, the interviews focused on eliciting participants' opinions that were linked to interruption occurrences that happened in the near past.

\subsection{Study Design and Analysis}

A total of 5 developers and 7 researchers ( 9 male, 3 female; age 28 - 45) from two industrial companies (7 and 5 persons per company) volunteered to participate in the study.
Company A is a large international producer of hardware and software for consumer electronics and medical products. Its research department is directly linked to the business lines and business managers are the clients for each research project. Researchers work in well-defined projects and under clear yet often quite long-term deadlines. The development departments of company A are directly dependent on business lines, also work on projects but under much shorter deadlines comparing to the research department. Company B is a large international producer of office hardware. Its research department is sponsored by the company and therefore the link between research projects and either development or business is much less pronounced comparing to company A. Researchers in company B have a lot of freedom to choose their own projects and areas of interest and are rarely working under the pressure of deadlines. Conversely, the development department of company B is much faster paced compared to the research department and also remains in direct dependence of business departments. However, as company B is predominantly focused on hardware development, the general pace of the projects is slower comparing to company A.

All participants were asked to describe their experiences with handling interruptions by means of semi-structured interview that addressed the following questions:

- Describe the last three face-to-face interruptions you experienced. How did they differ from other interruptions?

- Can you describe a recent face-to-face interruption which you decided to handle immediately although you were busy? How did it differ from other interruptions?

- Can you describe a recent face-to-face interruption that you decided to postpone although you were not that busy? How did it differ from other interruptions?

Interviews lasted about 60 minutes, were recorded and transcribed. Each statement was written on a post-it note, with an annotation whether it referred to an immediately handled or a postponed interruption. In this way, 178 statements were labeled. Next, an independent coder was asked to perform an open coding on the statements using Affinity Diagrams [42]. After distributing all statements into 6 clusters the coder described the characteristics of each cluster and named them. Next, the second coder coded all statements using categories derived by the first coder. He was encouraged to create his own clusters if the statements did not fit into the provided categories. Then, both coders met in a joint session to discuss the identified categories and let higher order relationships among them emerge in a form of a tree diagram [50] (see: Fig. 6). Finally, the definitions of each category and subcategory were formulated and exemplary quotes were selected ${ }^{2}$.

\subsection{Results}

Participants reported 56 occurrences of face-to-face interruptions: 41 that were immediately handled and 15 that

\footnotetext{
${ }^{2}$ This analysis was repeated by 3 teams of Master students from the Industrial Design department of Eindhoven University of Technology as a part of the Qualitative Research Methods course. Although their analysis was less rigorous, the results obtained by the student teams confirmed the outcome of the analysis presented in this article.
} 

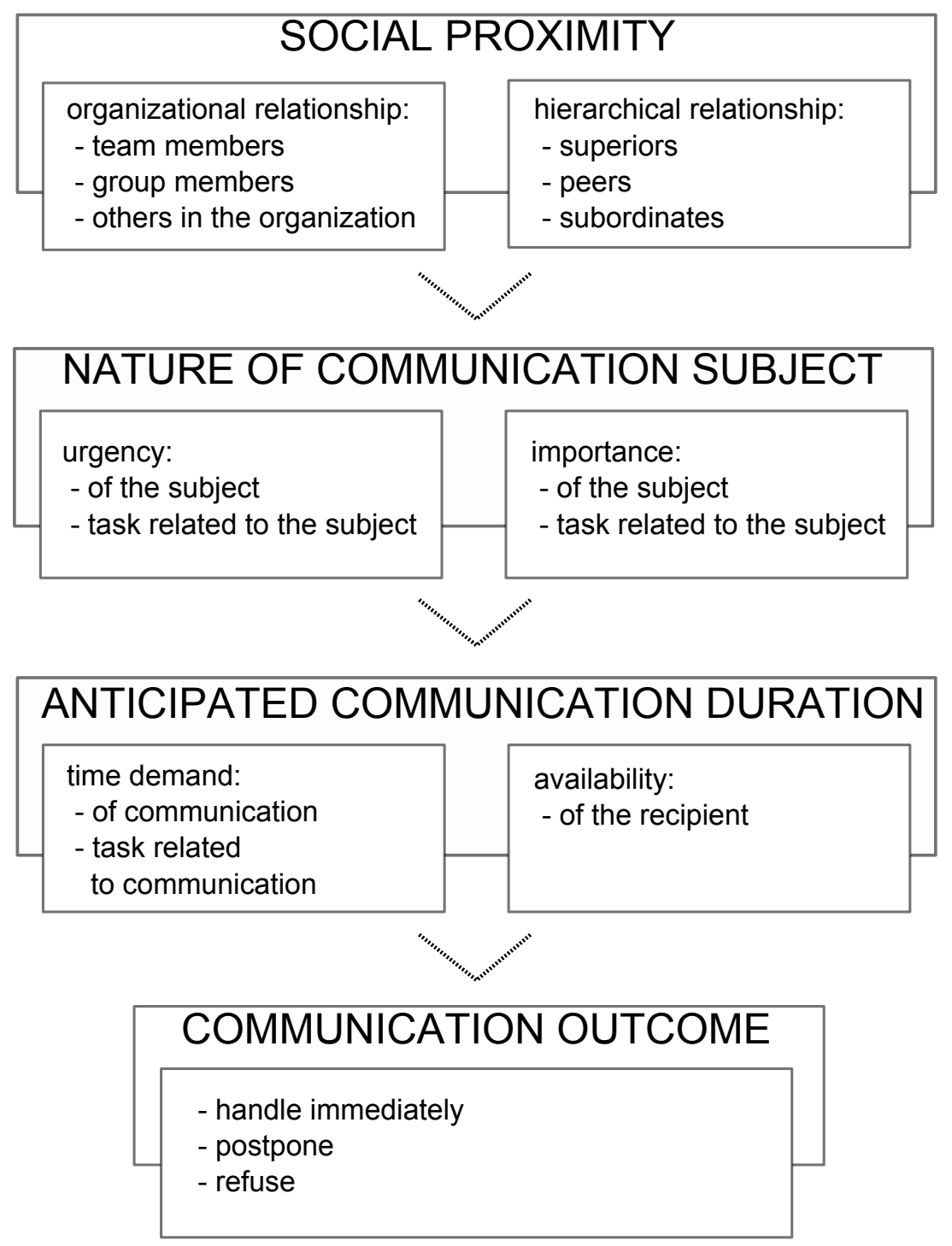

Fig. (6). An illustration of the relationships between the categories derived from the interview results analysis.

were postponed. The analysis of their statements resulted in the formulation of three categories depicting factors influencing the decision how to handle face-to-face interruptions: social proximity, the nature of the interruption subject and the anticipated interruption duration. Social proximity consisted of two subcategories: organizational and hierarchical relationship between communicators. The nature of the interruption subject was found to contain the following subcategories: urgency and importance of that subject. The anticipated communication duration was constructed out of two subcategories: interruption time demand and interruptee's availability state.

\subsubsection{Social Proximity}

Social proximity characterizes the professional relationship between communicators and was mentioned by all participants as a crucial motivator for deciding how to deal with face-to-face interruptions. Participants distinguished between two social dependencies: organizational and hierarchical. They discriminated organizational relations among:
- $\quad$ Team members - a relationship in which both actors share a common goal and collaborate together to achieve it;

- Group members - a relationship, in which both actors perform their tasks independently, while collaborating to achieve their own objectives;

- Other members of one's organization - a relationship, in which both actors perform their own tasks in total disconnection with each other's activities and goals.

Team members were likely to receive immediate attention regardless the interruption subject, all of which were motivated by sharing a common goal (27 immediately handled, 7 postponed). Granting attention to a group member or another person from the organization seemed to depend on participants' availability state ( 7 were immediately handled and 8 postponed).

'Who I usually allow to interrupt me right away is a person from my team. I work with them, so I assume that their questions are relevant to me as well.' (P3) 
A person from your group is one step further than your team. There is some sense of belonging but, in fact, he is not working with you directly that you don't interact that often. So, if he asks a question I have a bit less difficulty saying to him: 'Well, I am busy now, come another time'.' (P7)

Social proximity is further assessed according to hierarchical relationship between communicators that depicts the power distance between them. The higher the interruptor's hierarchical position, the more likely he or she was to receive participants' immediate attention. Such behaviour was detected in 7 cases and was motivated by the appreciation of superior's time and participants' eagerness to maintain a positive professional image.

'When my manager interrupted me I was really focused, so I didn't directly respond to him but asked: 'One second, I have to finish this one sentence". I finished the email I was writing and then I was all ears. If he comes it means that something is going on. The problem itself can even be trivial but he is also judging my work and I want to make a good impression.' (P1)

'You can't just refuse him (the boss) because he is a person who has little time and if he comes to you, you feel really involved. You have to be involved because it might be hard for him to come back later. And his decisions might have a high impact on my job.' (P8)

\subsubsection{The Nature of the Interruption Subject}

Once an interruptor arrived at the office, 8 out of 12 participants reported to always inquire about the reason for the interruption first before deciding whether to accept or postpone the interruption. In such a way they tried to assess its importance and urgency. Urgency pertains to how quickly the interruption should be handled and was assessed in terms of a deadline to the task that related to the interruption. Importance indicates the subjective value of the interruption subject to both communicators. It is crucial to note that urgency and importance level is not necessarily equal for the interruptor and the interruptee. In such a case participants tended to compare the importance and urgency of their own task with that of the interruption. Three participants chose to postpone an interruption to verify the importance of its subject (assuming that the interruptor would return if the issue was really important). Four participants decided to keep on postponing an interruption (which, in fact meant rejecting it) because its subject was of no interest to them.

'He tried to solve it himself and only after he couldn't handle it and it had to be ready for the next day, he came to me. So, I helped right away.' (P10)

'They came to my room and asked me to make a poster for them. I thought I was too busy to help them out. So, the discussion became, in fact, a negotiation between how important it was for them that I did it and also when it had to be finished. As it turned out really important and I was the only person who could do it, I decided to stay at work late and have it ready by the next morning.' (P3)

\subsubsection{Anticipated Interruption Duration}

The remaining four participants mentioned that they would begin with assessing the anticipated interruption duration when dealing with face-to-face interruptions.
Interruption duration pertained to the balance between the time they had to spend handling the interruption effectively and their own availability. Interruptions that required little time were often immediately handled (33 immediately handled, 6 postponed) while those perceived as long are more frequently postponed until participants could dedicate sufficient time for them (4 immediately handled, 13 postponed). The main reason behind immediately handling short interruptions was to prevent future commitments and to avoid having to remember about coming back to the interruptor. Participants felt that the cost of having a small break in their present task was usually lower than having to return to the interruptor at a later stage. Three participants reflected that a large number of interruptions, even if each one of them was rather short, caused a feeling that, with interruptions piling up, they fail to carry on their tasks.

'He came to my room and asked: 'Do you have 5 minutes for me? I have this small question'. If it is that short then my own availability doesn't really matter, then I can do it for you anyway. If it is longer than 5 minutes, then it comes in last after all the other things I have to do today, it goes into a cue.' (P9)

'It is not the number of interruptions but the time they take. Yesterday, I've got a very annoying interruption, it took whole day. He just kept me for so long that I didn't manage to get my own work done.' (P2)

\subsection{Discussion}

The results derived a set of factors influencing the availability state of knowledge workers and determining the decision regarding how to handle an interruption. Consistently with the study of assistants' interruption behaviours, also this study showed that the decision how to handle an interruption is based on its nature (with urgency and importance as attributes) and its anticipated duration (with time-demand as an attribute). Contrary to the results derived from the assistants' study, this study revealed social proximity between communicators to be an important motivator for deciding how to deal with an interruption. As earlier discussed, such a result could be explained by less profound relationships between an assistant and other workers comparing to the working relations among coworkers. Due to their job characteristics, assistants are rarely deeply entangled in collaborative activities that lead to one's professional success of failure. Therefore, they may be less sensitive to the organizational dependencies such as a team or group membership comparing to knowledge workers. They may also have a different perception regarding the hierarchical dependencies across the organization as they negotiate for their bosses the interruptions coming from important people (like other managers). Likewise, social proximity is more likely to have impact on interruption behaviours of knowledge workers due to multiple professional and private dependencies relating to past or future collaborations but also different levels of reciprocity.

Thus far a set of factors influencing the decision how to react to a face-to-face interruption was considered. We chose to examine face-to-face communications since they are considered the richest channel that supports an immediate feedback regarding communication subject and also for seamless negotiation of its duration [23, 51, 52] and 
therefore most likely to derive an extensive account of factors influencing interruption behaviours. A crucial difference between the face-to-face and mediated communication is the way the communication is negotiated [53]. Any mediated channel is, in its nature, empoverished comparing to face-to-face in terms of social cues [54] and therefore communication negotiation often appears more clumsy and graceless. Media of lower richness such as Instant Messaging or email tend to convey fewer cues regarding the context of communication and restricted feedback regarding the interruption content and timing [53]. On the other hand their uncertainty regarding the communication follow-up enables plausible deniability [55], supporting the interruptee in attending the communication subject at her own convenience. Lowering the richness of the communication channel might then show a strong impact on which factors are likely to impact on interruption behaviours. Therefore, a further understanding of the impact of the previously identified factors on the decision regarding how to handle mediated communications is likely to help identifying the ways to design support for communication negotiation. The goal of the next study was to examine what is the effect of social proximity, nature of the communication subject and anticipated interruption duration on the change in the availability for communication in email.

\section{KNOWLEDGE WORKERS AND HANDLING EMAILS}

In recent years email has become an integral means to communicate in both professional and private settings. It is composed out of a number of unique characteristics such as being asynchronous [20], textual [21], shared [56], traceable $[13,57]$, instantaneous [58] and effcient [22]. Email popularization brought about a large disadvantage: email overload. The feeling of email overload is caused, among other factors, by the fact that messages tend not to visually differ in importance, urgency, required effort and interest, which then needs to be deduced either from the message subject or directly from its content $[21,59,60]$. Hair et al. [61] argued that to reduce the level of stress induced by a large volume of newly arriving emails and to restore the control over this communication channel it is crucial to define and measure the 'orientations' towards email. An 'orientation' is an email characteristic that helps people to define whether to handle a particular message immediately, postpone or delete it. Factors such as social proximity, nature of the communication subject (thus its importance and urgency) and anticipated interruption duration can be perceived as such 'orientations'. The interplay between them and the interruptee's decision regarding email handling is examined in the section that follows.

\subsection{Study Design and Analysis}

A total of 10 persons ( 6 male, 4 female) agreed to record their email communications through a diary study for a period of one day. Diary studies show high ecological value as they are carried in situ [43]. On the negative side, they impose high burden on the participants as they require systematic recall of events. Furthermore, they run a risk of invoking a 'Heisenberg effect': participants' recalls could be influenced by the observing process itself [43]. Despite these disadvantages, diary studies allow for conducting analysis regarding factors influencing interruption behaviours in asynchronous communication based on ecologically valid data that is likely to give insights into patterns pertaining to dealing with emails. Each entry in the booklet was supposed to be entered at the exact moment of email arrival and consisted of the following information: time-stamp, email subject and interruptor's identifier. Furthermore, participants judged each email according to the following criteria:

- (5) very unavailable, (4) unavailable, (3) somewhat unavailable, (2) rather available, (1) available.

- To record email's urgency: How quickly, you felt, should this email be handled? - (5) immediately, (4) in the next 2-3 hours, (3) today, (2) this week, (1) whenever.

- To record email's importance: How important was it to handle the email immediately? - (5) very important, (4) important, (3) somewhat important, (2) rather unimportant, (1) unimportant.

- $\quad$ To record email's time demand: How much time, did you think, you had to dedicate to handle it? - (5) more than 1 hour, (4) about 1 hour, (3) about 10-30 minutes, (2) about 5-10 minutes, (1) less than $5 \mathrm{~min}$.

- To record social proximity between the participant and the email sender: What is your relationship with the sender?

- To record the action on email: Should this email be (1) immediately handled, (2) postponed or (3) deleted?

Participants were reminded every 2 hours about updating the booklet, so that data about emails was collected as close to their arrival as possible. Since the reminders were sent by email, participants were asked to discard these as data points. They were also asked to ignore messages coming from mailing lists and spam.

Each data point was inspected and 44 (out of 134) incomplete entries were removed. Such a surprisingly large number of incomplete data entries could be explained in two ways: Participants reported having received some emails which they considered to be junk messages in the process of their evaluation. Therefore, they did not attempt to finalize their description in the diary. The remaining incomplete data entries were explained by being distracted from filling in the diary by another person or activity. In such situations participants seemed to have forgotten to finalize the email evaluation. For the remaining 90 emails, the causal variables (the importance of the interruptor, urgency and importance of the email subject, time-demand of answering and availability of the interruptee) were first coded in the following way:

- Interruptors marked on the 6-point scale as either superiors or team members were coded as important, those marked as group members or other members of the organization were coded as unimportant. Finally, all professional or private external contacts were coded as external ${ }^{3}$.

\footnotetext{
${ }^{3}$ This category has been identified in an earlier study of Instant Messaging communications by Avrahami and Hudson [62]
} 
- Interruptions marked on the 5-point scale as either very important or important, were coded as important, while those marked as equally important, less important, or unimportant, were coded as unimportant.

- Interruptions marked on the 5-point scale as either to be immediately handled or to be handled in the next 2 hours, were coded as urgent, while those marked as to be handled today, this week, or whenever, were coded as not urgent.

- Interruptions marked on the 5-point scale as either requiring less than 5 minutes or about 5-10 minutes were coded as little time-demand, while those marked as requiring about 10-30 minutes, about 1 hour, or more than 1 hour, were coded as large time-demand.

- $\quad$ Availability state of the interruptee marked on the 5point scale as either available or rather available were coded as available, while those marked as slightly unavailable, rather unavailable or unavailable were coded as unavailable.

The binomial values together with confidence intervals were separately calculated for each causal variable and the dependent variables (i.e., interruption outcome).

\subsection{Results}

A total of 90 emails were recorded in the study: 53\% of them was immediately handled and $47 \%$ postponed or ignored. Participants were contacted by team members in $10 \%$ of cases $(n=8)$ and by group members in $8 \%$ of cases $(n=7) .23 \%$ of recorded messages came from participants' superiors $(n=21) .22 \%$ arrived from other people from their organization either geographically collocated or distributed $(n=20)$ and $38 \%$ from external contacts, both professional and private $n=34$, professional $(=22$, private $=12)$.

\subsubsection{Nature of the Email Subject}

In line with our expectations, the nature of the email subject proved to be a sufficient predictor of an action on email. The collected data showed that if the email subject was both important and urgent, it had higher probability to be immediately handled rather than to be postponed (see: Fig. 7). Surprisingly, also emails that were considered neither urgent nor important were more likely to be immediately handled than to be postponed.

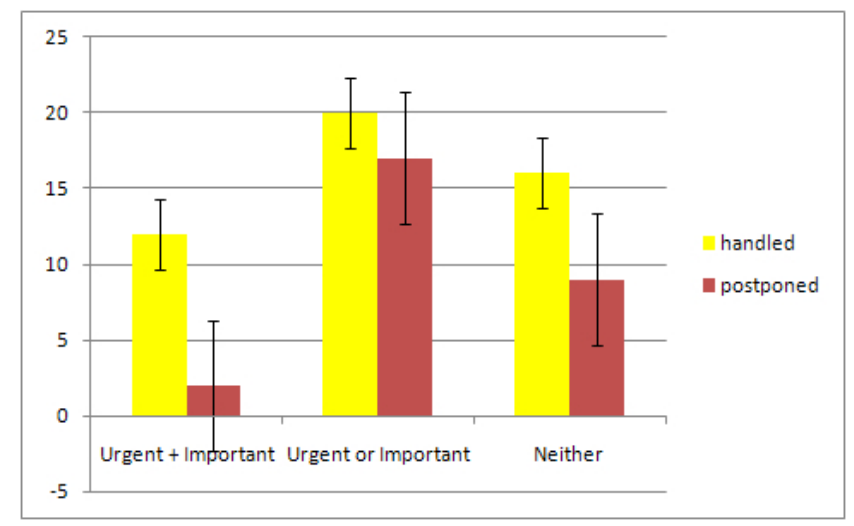

Fig. (7). The relative impact of the importance and urgency of the interruption subject on the interruptee's decision regarding an action on email.

\subsubsection{Anticipated Email Handling Duration}

The anticipated email handling duration also appeared to be a good predictor for an action on email. Emails with low time demand were likely to be immediately handled, while those considered as requiring a long time were more likely to be postponed (see: Fig. 8). The data further revealed that emails with a low time demand were likely to be immediately handled even if the interruptee appeared unavailable ( median $=3$ ). It was also noted that emails requiring no more than 10 minutes to be answered were frequently immediately handled although their subject was neither important ( median $=2$ ) nor urgent $($ median $=1.5)$.

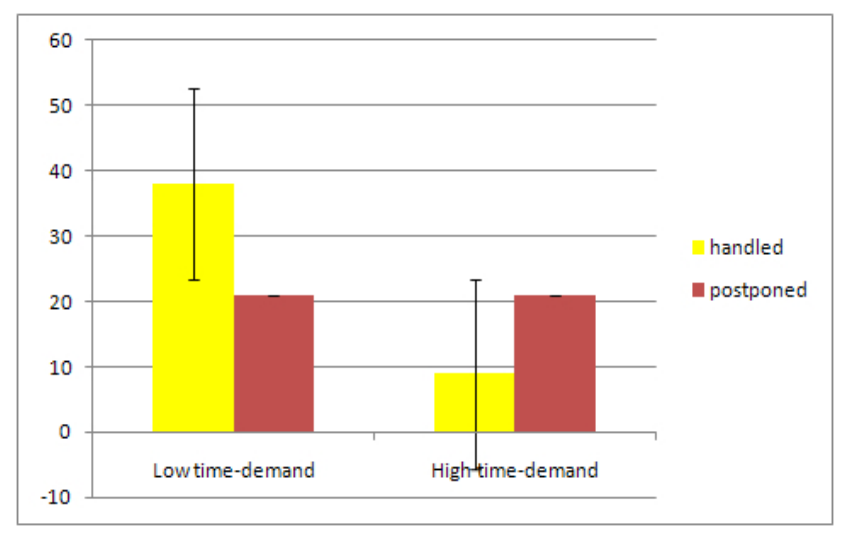

Fig. (8). The relative impact of the interruption time-demand on the interruptee's decision regarding an action on email.

\subsubsection{Social Proximity}

Social proximity was not a good predictor of an action on email. Important, less important people from participants' organization, as well as their external contacts, had almost the same chance of having their emails immediately answered as postponed. There was approximately a chance of $51 \%$ that emails from superiors, team members, group members as well as other people from the participants' organization would be immediately answered. Emails coming from external contacts had a $55 \%$ chance to be immediately handled.

\subsubsection{Availability of the Interruptee}

Similarly to the results of the study regarding the interruption handling behaviours of the assistants, also this study showed that the availability level was also not a good predictor of an action on email. An email had almost an equal chance of being immediately handled as postponed regardless of whether the interruptee was available or unavailable for email communication.

\subsection{Discussion}

The goal of this study was to explore the impact of factors found in the previous studies on interruption behaviours in asynchronous communication. We observed that, contrary to qualitative insights collected from knowledge workers regarding face-to-face communications (discussed in section 4.3), social proximity appeared to have little impact on an action on email. Emails coming from team members and superiors had similar chance to be immediately answered as to be postponed. The same was observed for emails coming from other people from one's 
organization and one's non-professional contacts. This result seems to contradict the findings of [63] who argued that the social relationship is an important predictor on an action on email.

The results showed that the anticipated handling duration could become a good predictor on an action on email. It might, therefore, be interesting to investigate ways to depict an anticipated email handling duration. For example, indicating email length (by displaying a number of lines) might appear indicative of the potential time demand required to write a response. The results also showed that emails considered as important and urgent had a high probability to be immediately handled. As previously stated, current email clients do little to help distinguishing among different emails. The results of this study imply that it might be valuable to provide additional metadata regarding email content. Displaying the urgency or the importance of the message (e.g., by marking a deadline to the task described in the message) might indicate to the recipient how quickly the response is desired and take away an implicit assumption that a sender expects an immediate response.

\section{GENERAL DISCUSSION AND CONCLUSIONS}

Many prior works have attempted to find means to suggest an 'appropriate moment' for an interruption [10, 24, $53,64,65]$. However, it seems that indicating availability prior to communication initiation does not always determine ensuing interruption behaviours $[47,64]$. In this article, three empirical investigations aimed at exploring the dynamic nature of the availability status and also investigating factors that influence that status. We further examined the impact of the identified factors on interruptees' decision regarding how to handle face-to-face and email communications. It was observed that any communication requiring little time had a large chance to be immediately handled regardless of the communication channel it was initiated through. Also communications considered as urgent and at the same time important were often immediately acted upon. Surprisingly, while social proximity was indicated to be a crucial factor for assessing face-to-face communications, it appeared to be an insufficient predictor for the action on email.

The results further suggest that factors such as social proximity, nature of the interruption subject and anticipated interruption duration influence and accordingly transform the availability state of the interruptee. Such a view on availability aligns with the definition of interpersonal privacy by Altman [66]:

'As a regulatory process, privacy can be viewed from two perspectives: a personally defined ideal level of interaction that a person or a group desires and a resulting outcome or achieved amount of actual interaction, which may or may not match what was desired.'

If assuming that the availability of an interruptee changes under the influence of the aforementioned factors, it becomes understandable why interruptors sometimes seem to neglect status indications about interruptee's availability [64]. As the interruptors knew they could influence the initial availability status, they carried on with the interruption attempting to weigh the respective importance of, for example, the interruption subject against the availability state of the interruptee. The analysis so far has shown that interruption negotiation is a dynamic process. Other authors have examined this negotiation as a collaborative effort to reach a mutual understanding about the communicative contract $[67,68]$. This argument is supported by the research reported in this article, to which we also contribute an account of the relevant factors that should be shared.

An initial illustration of factors influencing availability state emerges thus far (see: Fig. 9) which shows that in order to stimulate social behaviours in communication it is vital, not only to present an accurate state on the interruptee's availability, but also other information that helps both communicators successfully agree upon a communicative contract. The order of importance regarding each factor differs depending on the communication channel; while in face-to-face communication social proximity could be seen as having high impact on the recipient's interruption behaviour; in email, the anticipated communication duration and the nature of the communication subject become strong determinants regarding the interruption outcome.

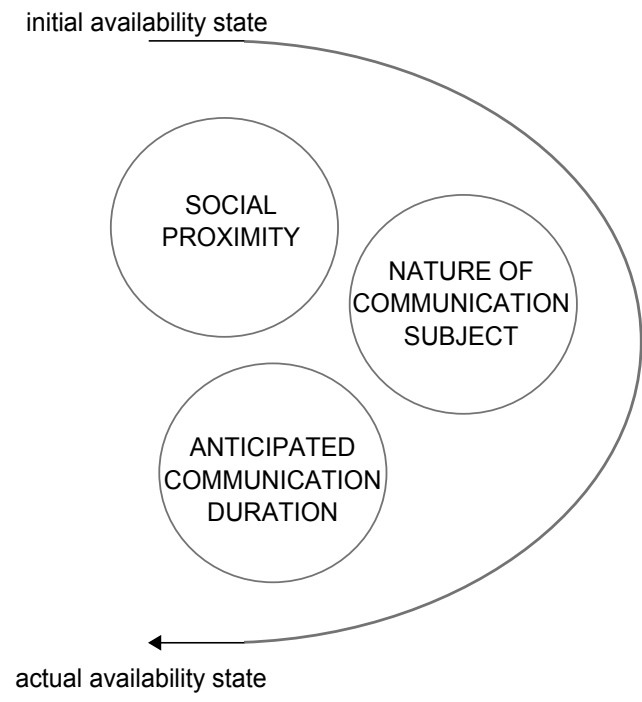

Fig. (9). The initial illustration of the availability state adaptation based on the work of Altman [66] fig. 1.1 (p.7) and fig. 9.4 (p.155).

Presenting such information is likely to shape the interruption negotiation, as both communicators gain the opportunity to provide feedback on the appropriateness of the interruption, which an interruptor might internalize and consider for future interruptions. Such feedback seems to be easier obtained when establishing communication through a rich and synchronous communication channel like face-toface. In such encounters both communicators have various means to express their needs and agree on how to best handle the communication subject. However, less rich communication channels like email do not support sharing of such awareness cues. Due to lack of awareness regarding the availability of the interruptee and the needs of the interruptor it becomes more difficult for communicators to agree on the most optimal communicative contract. For example, social proximity seems to have a large impact on synchronous 
communication, in which actors meet face-to-face, but it has far lesser influence on mediated email communications, where the physical presence does not regulate interplay between the actors. Nonetheless, we argue that in order to truly leverage social behaviours of people it is crucial to visualize the factors identified in these studies during the communication negotiation process (regardless of the fact whether the communication occurs in physical or digital world, and if it is synchronous or asynchronous in its nature).

\section{REFERENCES}

[1] Whittaker S, Frohlich D, Daly-Jones O. Informal workplace communication: What is it like and how might we support it? In: CHI. New York: ACM Press 1994; pp. 131-7.

[2] Heyer C, Brereton M. Socialising across channels: group multichannel communication. In: CHISIG 2006; pp. 421-4.

[3] Kraut RE, Fish RS, Root RW, Chalfonte BL. Informal communication in organizations: form, function, and technology. Human reactions to technology: Claremont symposium on applied social psychology 1990.

[4] Karim NSA, Heckman R. Group communication media choice and the use of information and communication technology to support learning: a case study. Campus-Wide Information Systems. 2005; 22(1): $28-42$.

[5] Su NM, Mark G. Communication chains and multitasking. In: CHI. New York: ACM Press 2008.

[6] Allen TJ, Hauptman $O$. The inuence of communication technologies on organizational structure: A conceptual model for future research. Information Technology and the Corporation of the 1990s: Research Studies 1994; pp. 475-83.

[7] Adamczyk PD, Bailey BP. If not now, when?: the effects of interruption at different moments within task execution. In: CHI. New York: ACM Press 2004; pp. 271-8.

[8] Kendon A. Conducting interaction: patterns of behavior in focused encounters. Cambridge University Press 1990.

[9] Trafton GJ, Monk CA. Task interruptions. Rev Hum Fact Ergon 2007; 3(1): 111-126.

[10] Begole J, Tang JC, Hill R. Rhythm modelling, visualizations and applications. In: UIST. New York: ACM Press 2003; vol. 1: pp. 11-20.

[11] Hudson JM, Christensen J, Kellogg TW, Erickson A. I'd be overwhelmed, but it's just one more thing to do. In: CHI. New York: ACM Press 2002; pp. 97-104.

[12] O'Conaill B, Frohlich D. Timespace in the workplace: dealing with interruptions. In: CHI Extended Abstracts. New York: ACM Press 1995; vol. 1: pp. 262-3.

[13] Clark H. Using language. vol. 1. $1^{\text {st }}$ ed. New York: Cambridge University Press 1996.

[14] Kakihara M, Sorensen C, Wiberg M. Fluid interaction in mobile work practices, the interaction society: practice, theories, and supportive technologies. In: The Interaction Society: Practice, Theories and Supportive Technologies. IDEA-group Inc. 2004.

[15] Oulasvirta A, Raento M, Tiitta S. ContextContacts: re-designing SmartPhone's contact book to support mobile awareness and collaboration. In: MobileHCI. New York: ACM Press 2005; pp. 167-74.

[16] Dabbish LA, Baker RS. Administrative assistants as interruption mediators. In: CHI Extended Abstracts ACM Press 2003; pp. 10201.

[17] Szostek MA, Markopoulos P. Factors defining face-to-face interruptions in the office environment. In: CHI Extended Abstracts. New York: ACM Press 2006.

[18] Gonzalez VM, Mark G. Managing currents of work: Multi-tasking among multiple collaborations. London: CSCW Springer 2005; vol. 1.

[19] Sproull LS. The Nature of managerial attention. In: advances in information processing in organizations. UK, Emerald Group Publishing Limited: JAI Press 1984; vol. 1: pp. 9-27.

[20] Thomas GF, King CL, Baroni B, et al. Reconceptualizing e-mail overload. J Bus Tech Commun 2006; 20(3): 252.

[21] Tyler JR, Tang JC. When can I expect an email response? a study of rhythms in email usage. In: CSCW Kluwer Academic Publishers 2003; pp. 239-58.
[22] Renaud K, Ramsay J, Hair M. "You've got e-mail!"... shall i deal with it now? electronic mail from the recipient's perspective. Int $\mathrm{J}$ Hum Comput Interact 2006; 21(3):313-32.

[23] Nardi B, Whittaker S. The place of face-to-face communication in distributed work. In: Hinds P, Kiesler S, eds. Distributed Work. MIT Press 2001.

[24] Wiberg M, Whittaker S. Managing availability: supporting lightweight negotiations to handle interruptions. ACM Trans Comput Human Interact 2005; 1(12): 356-87.

[25] Patil S, Lai J. Who gets to know what when: configuring privacy permissions in an awareness application. In: CHI. New York: ACM Press 2005; vol. 1: pp. 101-11.

[26] Bailey BP, Konstan JA, Carlis JV. On the Need for AttentionAware Systems: Effects of Interruption on Task Performance, Error Rate, and Affective State. J Comput Human Behav 2005; 1(special issue on attention aware systems).

[27] Speier C, Valacich JS, Vessey I. The effects of task interruption and information presentation on individual decision making. In: Proceedings of the eighteenth international conference on Information systems. Association for Information Systems 1997; pp. 21-36.

[28] Speier C, Vessey I, Valacich JS. The effects of interruptions, task complexity, and information presentation on computer-supported decision-making performance. Decis Sci 2003; 34(4): 771-97.

[29] Dabbish L, Kraut R. Controlling Interruptions: Awareness Displays and Social Motivation for Coordination. In: CSCW. New York: ACM Press 2004; vol. 1: pp. 182-91.

[30] Romero N, Szostek Matysiak A, Kaptein M, Markopoulos P. Behaviours and preferences when coordinating mediated interruptions: social and system inuence. In: ECSCW. New York: Springer 2007; pp. 351-70.

[31] Gonzalez VM, Mark G. Constant, constant, multi-tasking craziness": managing multiple working spheres. In: CHI. vol. 1 ACM Press 2004; pp. 113-20.

[32] Latorella KA. Investigating Interruptions: Implications for Flightdeck Performance. State University of New York at Buffalo 1996.

[33] Bailey BP, Konstan JA. On the need for attention-aware systems: Measuring effects of interruption on task performance, error rate, and affective state. Comput Hum Behav 2006; 22(4): 685-708.

[34] Goffman E. Interaction ritual: essays in face-to-face behavior. Random House Inc 1967.

[35] Brown P, Levinson SC. Politeness: Some Universals in Language Usage. Cambridge: Cambridge University Press 1987.

[36] Erickson T, Danis CM, Kellogg WA, Helander ME. Assistance: the work practices of human administrative assistants and their implications for it and organizations. In: CSCW. ACM Press 2008; pp. $609-18$

[37] Beyer H, Holtzblatt K. Contextual design: defining customercentered systems. San Francisco, CA: Morgan Kaufmann Publishers Inc. 1998.

[38] Kahneman D, Krueger AB, Schkade DA, Schwarz N, Stone AA. A survey method for characterizing daily life experience: the day reconstruction method. Science 2004; 306(5702): 1776-80.

[39] Goffman E. Strategic interaction. Oxford: Blackwell 1970.

[40] Fleiss JL, Levin B, Paik MC. Statistical methods for rates and proportions. NY, USA: Wiley New York; 1981.

[41] Strauss A, Corbin JM. Basics of qualitative research: grounded theory procedures and techniques. Thousand Oaks, CA: Sage Publications Inc. 1990.

[42] Hackos JT, Redish JC. User and Task Analysis for Interface Design. vol. 1. New York: John Wiley \& Sons 1998.

[43] Czerwinski M, Horvitz E, Wilhite S. A diary study of task switching and interruptions. In: CHI. New York: ACM Press 2004; vol. 1: pp. 175-82.

[44] Bailey BP, Konstan JA, Carlis JV. The e ects of interruptions on task performance, annoyance, and anxiety in the user interface. In: Hirose M, editor. Interact. vol. 1. Amsterdam, Netherlands: IOS Press 2001; p. 593-601.

[45] Iqbal ST, Bailey BP. Investigating the effectiveness of mental workload as a predictor of opportune moments for interruption. In: CHI. CHI Extended Abstracts. New York: ACM Press 2005; pp. 1489-92.

[46] Nardi BA, Whittaker S, Bradner E. Interaction and outeraction: instant messaging in action. In: CSWW. New York: ACM Press 2000; pp. 79-88. 
[47] Fogarty J, Lai J, Christensen J. Presence versus availability: the design and evaluation of a context-aware communication client. Int J Human Comput Stud 2004; 61(3): 299-317.

[48] Schwarz N, Kahneman D, Xu J, Belli R, Stafford F, Alwin D. Global and episodic reports of hedonic experience. Using calendar and diary methods in life events research. In press 2010.

[49] Stone AA, Schwartz JE, Schkade D, Schwarz N, Krueger A, Kahneman D. A population approach to the study of emotion: diurnal rhythms of a working day examined with the Day Reconstruction Method. Emotion 2006; 6(1): 139-49.

[50] Morse JM, Field PA. Qualitative Research Methods for Health Professionals. Thousand Oaks, CA: Sage Publications Inc. 1995.

[51] Daft RL, Lengel RH. Information richness: a new approach to managerial information processing and organizational design. Res Organ Behav 1984; 6: 191-234.

[52] Daft RL, Lengel RH. Organizational information requirements, media richness and structural design. Manag Sci 1986; 32(5): 55471.

[53] Tang JC. Approaching and leave-taking: Negotiating contact in computer-mediated communication. ACM Trans Comput Hum Interact 2007; 14(1)

[54] Sproull L, Kiesler S. Reducing Social Context Cues: Electronic Mail in Organizational Communications. Manag Sci 1986; 32(11): 1492-512.

[55] Aoki PM, Woodruff A. Making Space for Stories: Ambiguity in the Design of Personal Communication Systems. In: CHI. ACM Press 2005; pp. 181-90.

[56] Dabbish LA, Kraut RE. Email overload at work: an analysis of factors associated with email strain. In: CSCW. New York: ACM Press 2006 pp. 431-40.

[57] Monk A. common ground in electronically mediated communication: clark's theory of language use. hci models, theories and frameworks: towards a multidisiplinary science. San Francisco, CA: Mogan Kaufmann Inc. 2003; pp. 265-89.

[58] Mackay WE. More than just a communication system: diversity in the use of electronic mail. In: CSCW. New York: ACM Press 1988; pp. 344-53.

[59] Whittaker S, Bellotti V, Gwiazdka J. Email in personal information management. Commun ACM 2006; 49(1): 68-73.

[60] Fisher D, Brush AJ, Gleave E, Smith MA. Revisiting Whittaker \& Sidner's email overload" ten years later. In: CSCW. New York: ACM Press 2006; pp. 309-12.

[61] Hair M, Renaud KV, Ramsay J. The inuence of self-esteem and locus of control on perceived email-related stress. Comput Hum Behav 2007; 23(6): 2791-803.

[62] Avrahami D, Hudson SE. Communication characteristics of instant messaging: effects and predictions of interpersonal relationships. In: CSCW. New York: ACM Press 2006; pp. 505-14.

[63] Neustaedter C, Brush AJB, Smith MA. Beyond" from" and" received": exploring the dynamics of email triage. In: CHI. ACM Press 2005; pp. 1977-80.

[64] Begole J, Matsakis NE, Tang JC. Lilsys: Sensing Unavailability. In: Press A, Ed. CSCW 2004; vol. 1: pp. 511-4.

[65] Fogarty J, Hudson SE, Atkeson CG, et al. Predicting human interruptability with sensors. ACM Trans Comput Hum Interact 2005; 12(1): 119-46.

[66] Altman I. The Environment and Social Behaviour: Privacy, personal space, territory, crowding. Monterey (Ca): Wadsworth 1975.

[67] Romero NA, Boer L, Markopoulos P. Interactive and lightweight mechanisms to coordinate interpersonal privacy in mediated communication. In: INTERACT. New York: Springer Verlag 2009.

[68] Romero NA, Markopoulos P. Grounding interpersonal privacy in mediated settings. In: GROUP. ACM Press 2009; pp. 263-72.

(C) Szóstek et al.; Licensee Bentham Open.

This is an open access article licensed under the terms of the Creative Commons Attribution Non-Commercial License (http: //creativecommons.org/licenses/ by-nc/3.0/) which permits unrestricted, non-commercial use, distribution and reproduction in any medium, provided the work is properly cited. 